\title{
Myenteric plexuses atrophy in the vicinity of colorectal cancer tissue is not caused by apoptosis or necrosis
}

\author{
*Anna Kozlowska ${ }^{1}$, ${ }^{\text {Przemyslaw Kwiatkowski }}{ }^{2}$, Agnieszka Oponowicz ${ }^{1}$, \\ Mariusz Majewski ${ }^{1}$, Zbigniew Kmiec ${ }^{2,3}$, Janusz Godlewski ${ }^{2}$
}

${ }^{1}$ Department of Human Physiology, Faculty of Medical Sciences, University of Warmia and Mazury, Olsztyn, Poland

${ }^{2}$ Department of Human Histology and Embryology, Faculty of Medical Sciences, University of Warmia and Mazury, Olsztyn, Poland

${ }^{3}$ Department of Histology, Medical University of Gdansk, Gdansk, Poland

*Both authors contributed equally

\begin{abstract}
Introduction. The previously performed studies showed that the presence of colorectal cancer (CRC) tumor is associated with the atrophy of myenteric plexuses in the vicinity of cancer invasion; however, the possible mechanisms of this phenomenon are not known. The aim of the present study was to determine whether the atrophic changes of the enteric nervous system (ENS) within an intestine wall of the CRC patients were caused by apoptosis or necrosis and whether they were associated with changes in the number of galanin-immunoreactive (GAL-Ir) neurons.

Material and methods. Samples of the large intestine wall located close to the CRC invasion and control, distally-located part of the colon, were collected from 9 CRC patients. The size of ENS plexuses and the number of neurons were compared. Triple immunofluorescent staining was used to visualize the co-expression of caspase 3 (CASP3) or caspase 8 (CASP8) with GAL and protein gene-product 9.5 (PGP 9.5, panneuronal marker) in the submucosal and myenteric ENS plexuses. The cells expressing myeloperoxidase (MPO, marker of neutrophils) and CD68 (marker of macrophages) were detected by immunohistochemistry around/in myenteric plexuses (MPs) and in the muscularis externa of the colon wall in the vicinity of tumor invasion.

Results. Myenteric plexuses in the vicinity of the CRC tissue were significantly smaller and had lower number of neurons per plexus than distantly located plexuses. The number of CASP8- and CASP3-Ir neurons in the ENS plexuses was similar in the colon wall both close to and distally from tumor invasion. The number of CASP8-Ir neurons within MPs located close to CRC invasion was higher than of CASP3-Ir neurons. The percentage of neurons co-expressing CASP8 and GAL in myenteric plexuses close and distantly from tumor was three-fold lower than of those co-expressing CASP3 and GAL. The mean number of neutrophils and macrophages inside and around myenteric plexuses located close to tumor invasion was higher or similar, respectively, as compared with adjacent muscularis externa.

Conclusions. The atrophy of myenteric plexuses in the vicinity of CRC invasion is not caused by apoptosis or necrosis. The differences in the proportions of neurons expressing galanin and the studied caspases suggest as yet unknown role of this neuropeptide in the mechanisms of neuron's atrophy in MPs located close to CRC tumor. (Folia Histochem Cytobiol. 2016, Vol. 54, No. 2, 99-107).
\end{abstract}

Key words: colorectal cancer; enteric nervous system; plexus; caspase 3; caspase 8; galanin; CD68; myeloperoxidase; IHC

Correspondence address: J. Godlewski, M.D., Ph.D.

University of Warmia and Mazury

al. Warszawska 30, 10-082 Olsztyn, Poland

e-mail: janusz350@poczta.onet.pl 


\section{Introduction}

The enteric nervous system (ENS) consists of myenteric plexuses (MPs), accumulation of neurons located between two layers of the muscularis externa, which are responsible for muscle contraction and proper peristalsis of the intestine, and three significantly smaller submucosal plexuses (SP) which regulate intestinal absorption and secretion. These are classified into inner SP (ISP) located close to the lamina muscularis mucosae, intermediate SP (IMSP) and the outer SP (OSP), situated near the circular layer of muscular externa [1].

Galanin (GAL) is a 30-amino-acid peptide which functions as a neurotransmitter not only within the central nervous system (CNS) but also in the human ENS including the myenteric and submucosal plexuses [2]. Within ENS galanin evokes contraction of the muscularis externa of the intestine and regulates intestinal peristalsis [3]. In our recent study, we have found higher GAL content in tissue homogenates of the parts of colon wall sampled from the area close to CRC infiltration than from the cancer-uninvolved part of the colon [4]. The study of proliferative enteropathy in pig demonstrated a notable increase in the number GAL-immunoreactive (GAL-Ir) neurons in the intramural ENS plexuses of the ileum wall [5]. Other studies involving transection (axotomy) of descending colon's external innervations showed a significant galanin increase within neurons of the sympathetic ganglia supplying this part of the large intestine [6]. Comparison of axotomy vs. acute inflammation of the intestine revealed that colitis caused a higher increase of GAL-Ir neurons number in the MPs of the porcine descending colon [7]. Thus, in many models of intestinal pathology galanin content and the number of GAL-Ir neurons may be altered suggesting the adaptive possibilities of the enteric neurons. This may be associated with the neuroprotective action of galanin that was well-documented in many experimental models of brain, nerve and neurons' injury [3, 8-10].

Our earlier studies regarding morphological changes of the ENS in human colorectal cancer (CRC) invasion, revealed that the MPs are atrophic in the vicinity of the tumor as compared with the unchanged (tumor-uninvolved) part of the colon wall $[4,11]$. However, to our best knowledge, there are no publications available to date which explain the mechanisms leading to this phenomenon. One of the causes of plexus atrophy may be neuronal apoptosis. Besides CNS [12] in the ENS apoptosis of neurons was observed in the colon in patients from severe constipation [13]. It is well-known that apoptosis may be activated by external or internal pathways. The external pathway is triggered by cell membrane receptors and then by activation of caspase 8 (CASP8). The internal pathway is initiated by cellular stress factors which cause cytochrome $\mathrm{C}$ to be released from the mitochondria, followed by the caspase 9 activation. Both of these pathways coincide at a common point, which is activation of caspase 3 (CASP3, 'executioner' caspase) and, from this moment, irreversible protein proteolysis and DNA damage progress in the cell [14].

We have assumed that ENS atrophic changes within the large intestine in the vicinity of cancer invasion site may be caused by the activation of the apoptotic pathway and/or necrosis. To test this hypothesis, a triple immunofluorescent staining in order to detect either CASP8 and GAL or CASP3 and GAL presence in the ENS neurons was performed. Furthermore, immunohistochemical staining was used to investigate the potential accumulation of inflammatory cells neutrophils and macrophages in the vicinity of MPs located close to cancer mass. The obtained results suggest that neither apoptosis nor necrosis are involved in the atrophy of myenteric plexuses of the ENS observe in the colon wall close to the CRC invasion.

\section{Material and methods}

Patient recruitment and material collection. The present study was conducted using post-operative material derived from 9 patients with CRC diagnosis. Post-operative pathomorphological analyses confirmed that patients included in this study formed a homogenous group with the same degree of adenocarcinoma invasion within the colonic wall, defined as T3 on the TNM scale by the American Joint Committee on Cancer (AJCC) staging. The tissue samples were collected during surgery at the Department of Oncological Surgery of the Regional Oncological Center in Olsztyn, Poland. The protocol of this study was approved by the University of Warmia and Mazury Bioethics Commission (No. 12/2012) and written informed consent was obtained from all patients in the study. All patients, five women and four men, had no evidence of obstruction or other disorders in the passage of bowel contents. Moreover, they did not have a second serious illness or neo-adjuvant chemo- or/ /and radiotherapy. The mean age of the patients was $66.78 \pm$ 9.73 years (range: $52-78$ years).

Directly after surgical resection of the intestine, small samples $(10 \mathrm{~mm} \times 10 \mathrm{~mm})$ were collected for immunohistochemical analyses from the removed part of the intestine. Samples were taken from the margin of cancer invasion (cancer-infiltrated intestinal wall) and from a macroscopically-unchanged segment of the large intestine wall at a distance of $5-8 \mathrm{~cm}$ from the tumor, as a control tissue. The tissue samples were then fixed in $4 \%$ buffered (pH 7.4) formaldehyde for $120 \mathrm{~min}$, washed in $0.1 \mathrm{M}$ phos- 
Table 1. Characteristics of the antibodies used in the study for triple-immunofluorescence and immunohistochemical staining

\begin{tabular}{|c|c|c|c|c|}
\hline Antisera & Code & Host species & Dilution & Supplier \\
\hline \multicolumn{5}{|c|}{ Triple-immunofluorescence staining of CASP 8, CASP 3, GAL and PGP 9.5 presence in neurons } \\
\hline \multicolumn{5}{|l|}{ Primary antibody } \\
\hline Active caspase 3 & ab2302 & Rabbit & $1: 1500$ & Abcam, Cambridge, UK \\
\hline Caspase 8 & $\mathrm{ab} 25901$ & Rabbit & $1: 1000$ & Abcam, Cambridge, UK \\
\hline Galanin & T-5034 (GHC 7100) & Guinea pig & $1: 1200$ & Bachem AG, Bubendorf, CH \\
\hline PGP 9.5 & $7863-2004$ & Mouse & 1:950 & Biogenesis, Kingstone, NH, USA \\
\hline \multicolumn{5}{|l|}{ Secondary antibody } \\
\hline Polyclonal anti-rabbit & E0432 & Goat & $1: 1000$ & Dako, Glostrup, DK, \\
\hline $\begin{array}{l}\text { Fluorescein (FITC) AffiniPure F(ab') }{ }_{2} \text { Fragment } \\
\text { Anti-Guinea Pig IgG }(\mathrm{H}+\mathrm{L})\end{array}$ & 706-096-148 & Donkey & $1: 450$ & $\begin{array}{l}\text { Jackson Immunoresearch, } \\
\text { West Grove, PA,USA }\end{array}$ \\
\hline $\begin{array}{l}\left.\text { AMCA AffiniPure } \mathrm{F}(\mathrm{ab})_{2}\right)_{2} \text { Fragment } \\
\text { Anti-Mouse } \operatorname{IgG}(\mathrm{H}+\mathrm{L})\end{array}$ & $715-156-151$ & Donkey & $1: 75$ & $\begin{array}{l}\text { Jackson Immunoresearch, } \\
\text { West Grove, PA, USA }\end{array}$ \\
\hline $\mathrm{Cy}^{\mathrm{TM}}$ 3-conjugated streptavidin & 016-160-084 & & 1:4500 & $\begin{array}{l}\text { Jackson Immunoresearch, } \\
\text { West Grove, PA, USA }\end{array}$ \\
\hline \multicolumn{5}{|c|}{ Immunohistochemical staining of macrophages and neutrophils } \\
\hline anti-CD68 & ab125212 & Rabbit & $1: 500$ & Abcam, Cambridge, UK \\
\hline anti-MPO & ab45977 & Rabbit & $1: 1000$ & Abcam, Cambridge, UK \\
\hline
\end{tabular}

phate buffer for three days, stored in $18 \%$ sucrose (in $0.1 \mathrm{M}$ phosphate buffer) for 7 days at $4^{\circ} \mathrm{C}$ and then stored at $-28^{\circ} \mathrm{C}$ until sectioning.

Immunofluorescence. Triple-labeling of PGP 9.5, galanin and caspase 8 or caspase $3.10 \mu \mathrm{m}$-thick cryostat (HM525 Zeiss, Germany) sections of intestine wall were subjected to a triple-immunofluorescence staining technique, using antibodies listed in Table 1, to detect: protein gene-product 9.5 (PGP 9.5; a pan-neuronal marker to visualize all neurons within plexuses) and caspase 3 (CASP3), caspase 8 (CASP8) and galanin (GAL) presence within the neurons. The sections were air-dried at a room temperature (RT) for $45 \mathrm{~min}$ and rinsed $(3 \times 15 \mathrm{~min})$ with phosphate buffered saline (PBS; pH 7.4). Afterwards, sections were blocked for $1 \mathrm{~h}$ at RT with a blocking solution consisting of $1 \%$ Triton X100 (Sigma-Aldrich, St. Louis, MO, USA), 0.1\% bovine serum albumin (Sigma-Aldrich), $0.05 \%$ thimerosal (Sigma-Aldrich), $0.01 \% \mathrm{NaN}_{3}$ (POCH, Gliwice, Poland) and $10 \%$ normal goat serum (Jackson ImmunoResearch, West Grove, PA, USA) in 0.01M PBS to reduce non-specific background staining. After washing in PBS, the mixture set of primary antibodies: PGP 9.5, CASP3, and GAL as well as PGP 9.5, CASP8, and GAL were added to the sections and the sections were incubated overnight in a humid chamber at RT. Following subsequent rinsing in PBS $(3 \times 15 \mathrm{~min})$, a mixture of was then added and the sections were incubated with secondary antibodies in a humid chamber at RT for 60 min. Subsequently, sections were washed in PBS $(3 \times$ $15 \mathrm{~min}$ ), and then cover-slipped with carbonate-buffered glycerol ( $\mathrm{pH}$ 8.6). The specificity of primary antisera was tested as follows: sections were incubated with an antibody that had been pre-absorbed with synthetic antigen $(10 \mu \mathrm{g}$ of antigen per $1 \mathrm{~mL}$ of diluted antiserum); the primary antibody was omitted from the incubation; or normal rabbit, guinea pig or mouse serum was substituted for the primary antibody.

Triple-immunolabeled neurons were analyzed under an Olympus BX61 microscope (Olympus, Tokyo, Japan) equipped with the epi-fluorescence kit and appropriate filter sets for AMCA (V1 module, excitation range 330-385 nm and barrier filter at $420 \mathrm{~nm}$ ), FITC (B1 module, excitation filter 450-480 nm) and CY3 (G1, excitation filter 510-550 nm). Images were taken using a CDD camera and cellSens Dimension $^{\mathrm{TM}}$ software (Olympus).

Immunohistochemical staining of macrophages and neutrophils. Cryostat sections of the intestine wall were prepared as described above and immunohistochemical (IHC) staining was performed using the method described by Jana et al. [15]. The samples were stained using an antibody against the cluster of differentiation 68 (CD68) as a macrophage marker or myeloperoxidase (MPO) as a neutrophil marker (antibodies listed in Table 1). Diaminobenzidine tetrahydrochloride (Sigma-Aldrich) was used as chromogen. The slides were then counterstained with Mayer's hematoxylin for $2 \mathrm{~min}$. The sections were analyzed and photographed using a light microscope (BX-41, Olympus, Tokyo, Japan) and XC-50 camera (Olympus) under $\times 200$ magnification (the area of a view field was $142,899 \mu \mathrm{m}^{2}$ ). 
Table 2. Mean area and number of neurons of the enteric nervous system plexuses in the colon wall in the vicinity of and distally from tumor tissue

\begin{tabular}{|c|c|c|c|c|c|c|}
\hline \multicolumn{3}{|c|}{ Mean plexus area $\left(\mu \mathbf{m}^{2)}\right.$} & \multicolumn{3}{c|}{ Mean number of neurons/plexus } \\
\hline $\begin{array}{c}\text { Close to tumor } \\
\text { invasion }\end{array}$ & $\begin{array}{c}\text { Distantly from } \\
\text { tumor }\end{array}$ & $\boldsymbol{p}$ & $\begin{array}{c}\text { Close to tumor } \\
\text { invasion }\end{array}$ & $\begin{array}{c}\text { Distantly from } \\
\text { tumor }\end{array}$ & $\boldsymbol{p}$ \\
\hline \multicolumn{7}{|c|}{ Myenteric plexus } \\
\hline $27786 \pm 24286$ & $46006 \pm 33961$ & $\mathbf{0 . 0 0 0 0 8}$ & $10.41 \pm 6.69$ & $24.34 \pm 17.15$ & $\mathbf{0 . 0 0 0 0 0}$ \\
\hline \multicolumn{7}{|c|}{ Outer submucosal plexus } \\
\hline $7561 \pm 6532$ & $4561 \pm 2445$ & 0.091 & $2.80 \pm 1.91$ & $4.50 \pm 2.13$ & 0.499 \\
\hline \multicolumn{7}{|c|}{ Inner submucosal plexus } \\
\hline $6324 \pm 8682$ & $3450 \pm 7789$ & $\mathbf{0 . 0 0 0 1 6}$ & $4.13 \pm 2.35$ & $4.09 \pm 2.29$ & 0.689 \\
\hline
\end{tabular}

The area of ENS plexuses was outlined according to the location of protein gene-product 9.5-immunostained neurons. Morphometry was performed as described in Material and methods. Data present an arithmetic mean \pm SD. P values of statistically significant differences between compared areas of the colon wall are shown in bold. Close to tumor invasion: sections of colon wall in an area adjacent to tumor tissue.

Morphometric analyses. The area of plexuses and number of neurons expressing PGP 9.5, galanin and CASP8 or CASP3. In each of the nine patients, in each of the two parts of the large intestine wall (located close to and distantly from tumor infiltration) cryostat sections (10 $\mu$ m thick), which were separated by a $200 \mu \mathrm{m}$ distance, were analyzed. In sections of tumor-adjacent areas of the human large intestine wall and in the control, tumor-uninvolved part three hundred of PGP 9.5-immunooreactive neurons were identified. The number of neurons co-expressing CASP 3 and GAL or CASP8 and GAL within myenteric plexuses as well as in OSP, IMSP and ISP was counted. The numbers of all neurons present in the OSPs, IMSPs and ISPs of the submucosa were added and presented as a total number of neurons in the submucosal plexuses.

The sections stained by triple-immunofluorescence method were studied under an Olympus BX61 microscope (Olympus, Tokyo, Japan), and were analyzed using the Cell program (Olympus). The area of the myenteric and submucosal OSP and ISP plexuses was measured and expressed in $\mu \mathrm{m}^{2}$, and the number of all PGP 9.5-Ir neurons in the plexuses was counted and expressed as mean per plexus.

The number of macrophages and neutrophils inside and around myenteric plexuses and in muscularis externa. From every patient 2 cryostat sections of colon wall obtained from the vicinity of cancer invasion ( $10 \mu \mathrm{m}$ thickness and separated at a $200 \mu \mathrm{m}$ distance) were analyzed. The number of CD68 positive cells (macrophages) and MPO-positive cells (neutrophils) was determined by overlaying the computer screen on which the photomicrograph was displayed with a film on which an ellipse of the size of $80 \times 160 \mu \mathrm{m}$ and area of $10,048 \mu \mathrm{m}^{2}$ at the same microscopic magnification was drawn. The ellipse covered the area including myenteric plexus or muscularis externa visible in the same microscopic view field at a distance of 226-320 $\mu \mathrm{m}$ from MP. The number of immunoreactive cells was counted inside the ellipse inside/ /around each MP as well as inside the ellipse that was placed over muscularis externa.

Statistical analysis. A Wilcoxon matched pairs test was used to statistically analyze the differences: the area of plexuses and number of neurons present in the vicinity of the cancer invasion site and the control part of colonic wall, comparison between immunoreactive neurons subgroups in SP and MP located close to the cancer mass and in the control sections. This test was used also to evaluate the inflammatory cell presence within MP area. In all performed analyses, the results were considered as statistically significant $(p<0.05)$.

\section{Results}

\section{Morphometry of myenteric and submucosal nervous plexuses in the vicinity of and distantly from CRC invasion}

The morphometric analysis of PGP 9.5-immunostained neurons revealed atrophy of myenteric plexuses in the vicinity of the CRC tissue estimated from significantly smaller size and lower number of neurons per plexus when compared with the plexuses located in colon wall distant from tumor invasion (Table 2). The size of outer submucosal plexuses and number of their neurons in both compared areas did not differ. The inner submucosal plexuses were significantly larger in the vicinity of cancer tissue than in the distant part of the colon wall while the number of neurons was similar in both compared regions (Table 2).

\section{The presence of CASP3-, CASP8- and galanin- -immunoreactive neurons in the ENS plexuses} in the vicinity of and distantly from CRC invasion The triple-labeling immunofluorescent staining revealed the differentiated pattern of neurons co-expressing CASP8 and GAL or CASP3 and GAL within 

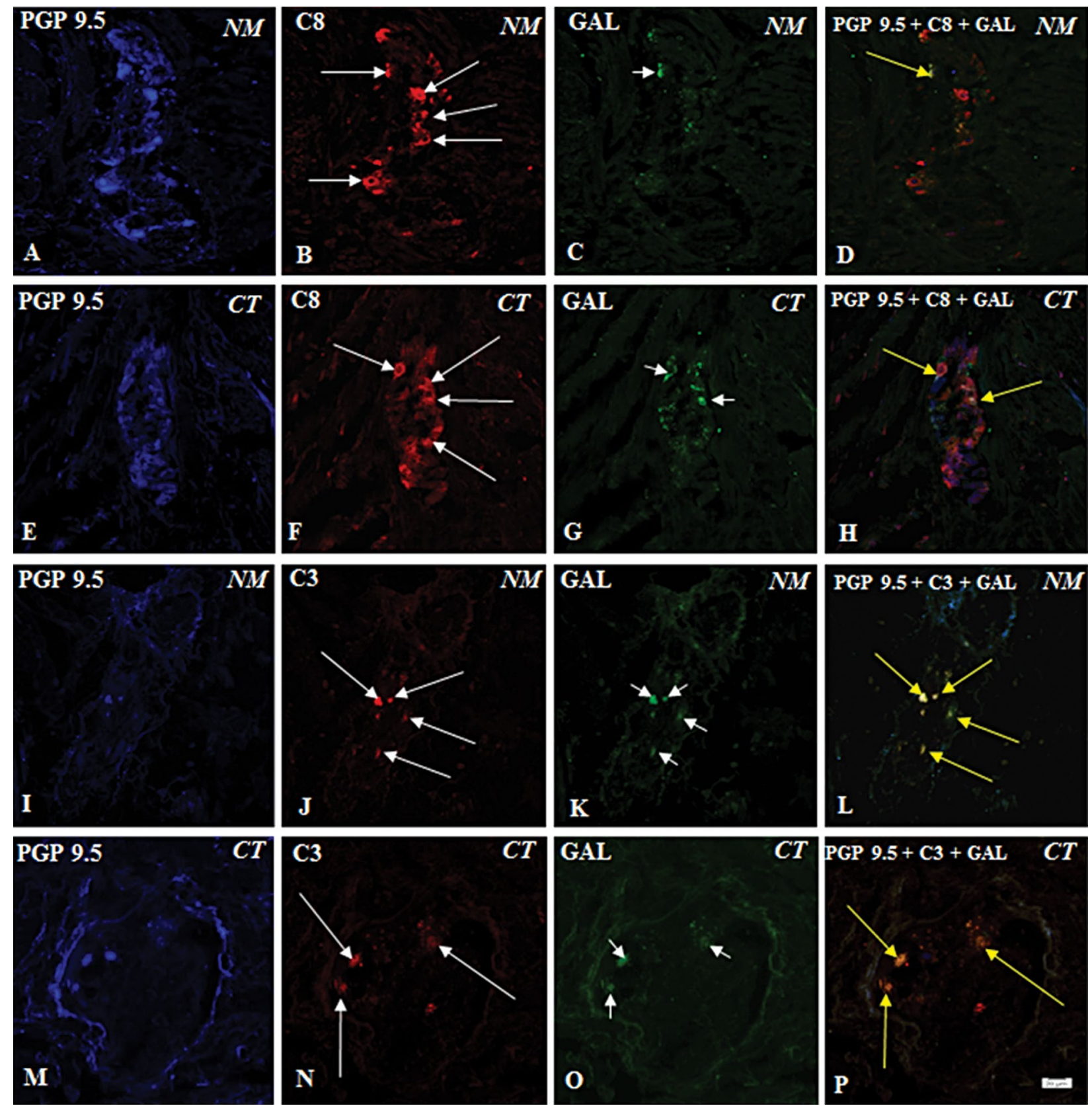

Figure 1. The representative images of protein gene-product 9.5 (PGP 9.5), caspase 8- (C8), caspase 3- (C3) and galanin(GAL) positive neurons located in myenteric plexus (MP) of human large intestine, in neoplasm margin $(N M)$ and in distally located part $(C T)$. The right side images $(\mathbf{D}, \mathbf{H}, \mathbf{L}$ and $\mathbf{P})$ are composed of the images taken from blue (A, E, I and $\mathbf{M})$, red $(\mathbf{B}, \mathbf{F}, \mathbf{J}$ and $\mathbf{N})$ and green $(\mathbf{C}, \mathbf{G}, \mathbf{K}$ and $\mathbf{O})$ fluorescent channels. All neurons present in MP visualized by PGP 9.5 staining (A, E, I and $\mathbf{M})$. C8+ (B and F) and C3+ $(\mathbf{J}$ and $\mathbf{N})$ neurons were marked by the long white arrows, GAL-positive neurons $(\mathbf{C}, \mathbf{G}, \mathbf{K}$ and $\mathbf{O})$ were pointed by the short white arrows. Triple immunoreactive $\mathrm{C} 8+/ \mathrm{GAL}+/ \mathrm{PGP} 9.5+$ neurons ( $\mathbf{D}$ and $\mathbf{H})$ and C3+/GAL+/PGP 9.5+ neurons ( $\mathbf{L}$ and $\mathbf{K})$ were matched by the long yellow arrows. Bar $20 \mu \mathrm{m}$.

myenteric (Figure 1) and submucosal (Figure 2) plexuses in the colon wall of CRC patients.

The proportion of CASP8-Ir neurons was four times higher in the myenteric vs. submucosal plexuses both in CRC-adjacent and distant part of the colon wall (Table 3). On the contrary, the proportion of
CASP3-Ir neurons was similar in the myenteric vs. submucosal plexuses in the CRC-adjacent part of the colon wall. However, in myenteric plexuses of the part of colon wall located distantly from CRC tissue the percentage of CASP3-Ir neurons was 2.5 times higher than in submucosal ones (Table 3 ). Similar proportion 

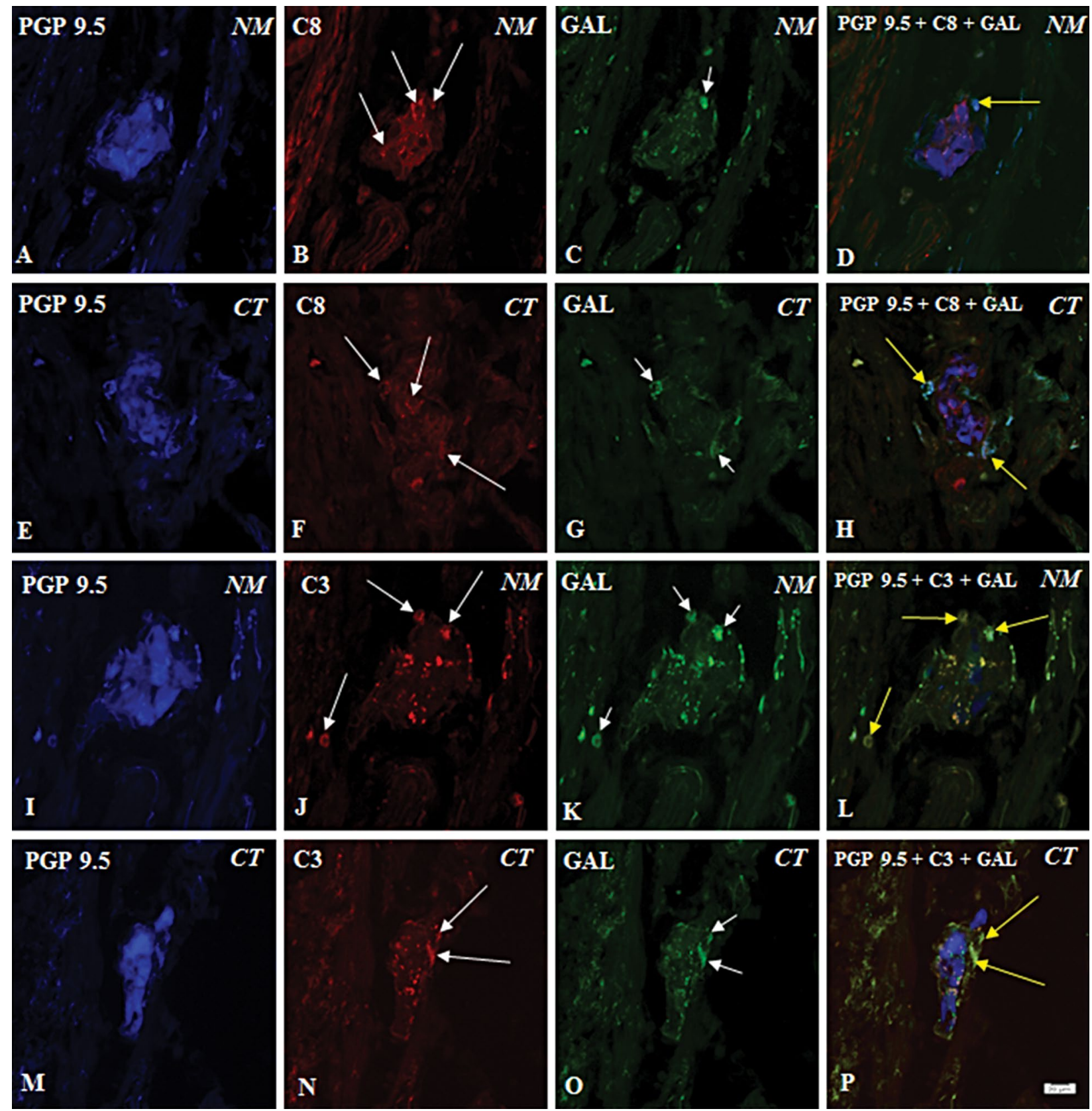

Figure 2. The images of intermediate submucosal plexus (ISP) and caspase 8- (C8), caspase 3- (C3) and galanin- (GAL) immunoreactive neurons present within them: from neoplasm margin $(N M)$ and in control part of intestine $(C T)$. The images (D, H, L and P) are compiled from images: blue (A, E, I and M), red (B, F, J and N) and green $(\mathbf{C}, \mathbf{G}, \mathbf{K}$ and $\mathbf{O})$. All neurons are visualized by PGP 9.5 staining (A, E, I and $\mathbf{M})$.

The long white arrows mark C8-Ir (B and F) and C3-Ir ( $\mathbf{J}$ and $\mathbf{N})$ neurons. The short white arrows mark GAL-positive neurons (C, G, K and O). Triple C8-Ir/GAL-Ir/PGP 9.5-Ir neurons (D and H) and C3-Ir/GAL-Ir/PGP 9.5-Ir neurons ( $\mathbf{L}$ and $\mathbf{K})$ were pointed by the long yellow arrows. Bar $20 \mu \mathrm{m}$.

of CASP3-Ir neurons was found in myenteric plexuses located close to and distantly from CRC invasion.

The proportion of all CASP8-Ir neurons within myenteric plexuses located close to CRC invasion did not differ from the percentage of all CASP3-Ir neurons within them $(p=0.109)$.
The comparison of myenteric and submucosal plexuses in relation to the percentage of neurons with the colocalization of CASP 3 and GAL or CASP8 and GAL revealed lack of differences in the both compared parts of the colon wall (Table 3). 
Table 3. The proportions of neurons with caspase 3 (CASP3) and galanin (GAL) or caspase 8 (CASP) and galanin colocalization in relation to the total number of protein gene-product 9.5 (PGP 9.5)-immunoreactive cells in myenteric and submucosal plexuses in the colon wall of CRC patients

\begin{tabular}{|c|c|c|c|c|c|c|}
\hline \multirow{2}{*}{$\begin{array}{c}\text { Neuron sub- } \\
\text { population } \\
\text { with studied } \\
\text { substances }\end{array}$} & \multicolumn{2}{|c|}{ Myenteric plexuses } & \multirow[t]{2}{*}{$p$} & \multicolumn{2}{|c|}{ Submucosal plexuses" } & \multirow[t]{2}{*}{$p$} \\
\hline & $\begin{array}{l}\text { Close to tumor } \\
\text { invasion }\end{array}$ & $\begin{array}{c}\text { Distantly from } \\
\text { tumor }\end{array}$ & & $\begin{array}{l}\text { Close to tumor } \\
\text { invasion }\end{array}$ & $\begin{array}{c}\text { Distantly from } \\
\text { tumor }\end{array}$ & \\
\hline \multicolumn{7}{|c|}{ Co-localization of caspase 3 and galanin (\%) } \\
\hline $\mathrm{CASP}^{+} / \mathrm{GAL}^{-}$ & $0.88 \pm 1.34$ & $2.06 \pm 4.61$ & 0.6831 & $0.1 \pm 0.2$ & $2.4 \pm 5.56$ & 0.2012 \\
\hline $\mathrm{CASP}^{+} / \mathrm{GAL}^{+}$ & $25.26 \pm 8.06$ & $34.39 \pm 17.66$ & 0.2056 & $22.1 \pm 24.8$ & $11.5 \pm 11.1$ & 0.3980 \\
\hline $\mathrm{CASP}^{-} / \mathrm{GAL}^{+}$ & $0.67 \pm 0.93$ & $1.37 \pm 1.69$ & 0.3477 & $11.6 \pm 14.3$ & $26.9 \pm 42.4$ & 0.0423 \\
\hline $\mathrm{CASP}^{-} / \mathrm{GAL}^{-}$ & $73.19 \pm 8.04$ & $62.11 \pm 16.52$ & 0.0663 & $66.2 \pm 28.7$ & $51.38 \pm 44.12$ & 0.5337 \\
\hline \multicolumn{7}{|c|}{ Co-localization of caspase 8 and galanin (\%) } \\
\hline $\mathrm{CASP}^{+} / \mathrm{GAL}^{-}$ & $37.41 \pm 15.86$ & $36.65 \pm 15.94$ & 0.8703 & $7.2 \pm 8.72$ & $11.46 \pm 15.92$ & 0.5286 \\
\hline $\mathrm{CASP}^{+} / \mathrm{GAL}^{+}$ & $8.64 \pm 9.0$ & $12.54 \pm 7.72$ & 0.1025 & $4.3 \pm 5.0$ & $3.06 \pm 4.34$ & 0.0506 \\
\hline $\mathrm{CASP}^{-} / \mathrm{GAL}^{+}$ & $3.25 \pm 2.57$ & $2.98 \pm 1.32$ & 0.9674 & $13.53 \pm 10.88$ & $13.03 \pm 17.09$ & 0.5541 \\
\hline $\mathrm{CASP}^{-} / \mathrm{GAL}^{-}$ & $39.59 \pm 18.41$ & $48.05 \pm 17.25$ & 0.5147 & $74.07 \pm 14.71$ & $72.44 \pm 26.17$ & 0.7897 \\
\hline
\end{tabular}

Morphometry was performed as described in Material and methods. Data presents an arithmetic mean \pm SD.

*Values obtained for inner and outer submucosal plexuses were summed up. $P$ values of statistically significant differences between compared areas of the colon wall are shown in bold.

However, the percentage of neurons co-expressing CASP8 and GAL in myenteric plexuses close to and distantly from tumor invasion was approximately three-fold lower than neurons co-expressing CASP3 and GAL (Table 3 ).

A comparison of GAL expression in CASP3-Ir and CASP8-Ir neurons in the submucosal plexuses showed higher $\mathrm{CASP}^{+} / \mathrm{GAL}$ co-expression than that of $\mathrm{CASP}^{+} / \mathrm{GAL}$ co-expression in the both analyzed parts of large intestine wall $(p=0.0280)$. It is of note, that almost all CASP3-Ir neurons in myenteric and submucosal plexuses in both studied parts of colon expressed galanin immunoreactivity (Figures 1 and 2, Table 3).

\section{The presence of neutrophils and macrophages in and around myenteric plexuses and adjacent muscle tissue}

Microscopic observations allow demonstrating that most of the mononuclear cells - neutrophils (MPO-immunoreactive cells) and macrophages (CD68-immunoreactive cells) were located at the periphery of and rarely within the plexuses (Figure 3A, C). On the contrary, the MPO-Ir and CD68-Ir cells were randomly distributed in the muscularis externa in the same microscopic view field (Figure 3B, D).

The morphometric analysis of the density of the studied mononuclear cells in the both analyzed areas (Figure 3) showed that the average number of neutrophils per the ellipsoid area (as described in Material and methods) was significantly higher around/in the myenteric plexuses than in muscle tissue present in the same microscopic view field (11.28 \pm 11.54 vs. $8.56 \pm$ 13.75 cells per $10,048 \mu \mathrm{m}^{2}$, respectively; mean $\pm \mathrm{SD}$, $p=0.0028)$. However, the cell density of macrophages was similar around/in myenteric plexuses and muscle tissue $\left(11.77 \pm 13.29 v s .11 .04 \pm 13.02\right.$, per $10,048 \mu \mathrm{m}^{2}$, respectively; $p=0.2774)$.

\section{Discussion}

Our study confirms the atrophy of myenteric but not submucosal ENS plexuses in the vicinity of the CRC invasion, and its results concur with our initial microscopic observations, which showed that changes in the morphology of MPs in the wall of large intestine were more evident as compared with SPs [11]. In a search for the mechanisms of this atrophy we decided to assess the possible role in this phenomenon of apoptosis or necrosis. Our observation of the higher proportion of CASP8-Ir neurons in myenteric plexuses in the vicinity of tumor tissue than in submucosal plexuses may suggest that observed atrophy is caused by apoptosis triggered by extracellular ligands. However, the observed similar percentage of CASP8 and CASP3-immunoreactive neurons in both compared parts of the large intestine does not support this assumption and indicates that atrophy of MPs is not caused by apoptosis.

Notwithstanding, the finding of higher number of CASP8-Ir neurons than CASP3-Ir neurons in 

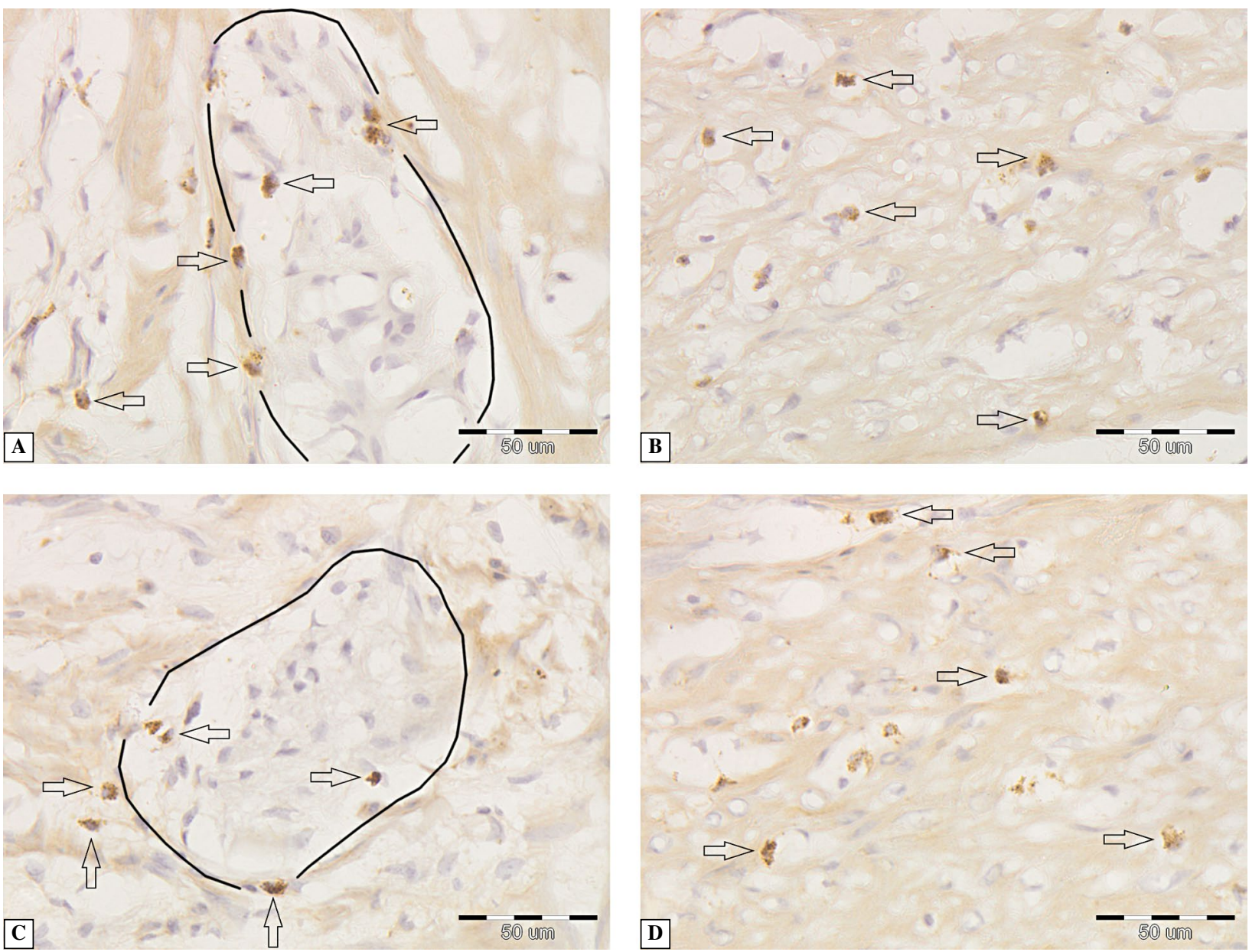

Figure 3. The presence of MPO-positive cells (neutrophils, arrows) around myenteric plexus (A) and in muscularis externa (B) and CD68-positive cells (macrophages, arrows) around myenteric plexus (C) and in muscularis externa (D). Sections obtained from a colon wall close to the CRC tumor tissue were stained by immunohistochemistry as described in Methods; bar $50 \mu \mathrm{m}$.

myenteric plexuses, may suggests silencing of the externally-activated apoptotic pathway during the effector phase of apoptosis. The prominent prevalence of neurons expressing CASP3 and GAL over the cells expressing CASP8 and GAL may suggest a possible role of galanin in the suppression of the apoptosis of ENS neurons. In an in vitro study it was shown that GAL or type $1 \mathrm{G}$ protein-coupled receptor galanin receptor (GALR1) silencing induced apoptosis in drug-sensitive and drug-resistant CRC cell lines. GALR1 silencing caused down-regulation of FLIP1, an endogenous CASP8 inhibitor, and consequently affected the activation of external pathway of apoptosis [16]. This putative apoptosis-protective role of galanin could represent another, as yet unrecognized, aspect of the neuroprotective function of this neuropeptide. However, it has to be noted that in some types of cancer cells, e.g. human head and neck squamous cell carcinoma (HNSCC) or rat pheochromocytoma
PC12 cell line, galanin was shown to be proapoptotic acting via its membrane GALR2 receptor [17].

The number of neutrophils (identified by the MPO immunoreactivity) was by $32 \%$ higher within/around myenteric plexuses than in surrounding smooth muscle tissue what may suggest that necrosis could be responsible by the myenteric plexuses' atrophy in the vicinity of CRC tumor invasion. However, we did not observe clear infiltration of mononuclear cells, which with a random distribution of macrophages (identified by CD68 immunoreactivity), does not support necrosis as a way of the observed atrophy of myenteric plexuses.

In an in vivo model (chorioallantoic membrane) of HNSCC metastases Scanlon et al. (2015) showed that galanin released by nerves initiated nerve-tumor crosstalk via activation of the GALR2 on cancer cells which resulted in the induction of galanin and cyclooxygenase-2 (COX-2) in tumor cells. The authors 
found that prostaglandin E2, an enzymatically derived product of COX-2 activity, promoted cancer invasion and, in a feedback mechanism, galanin released by cancer induced neuritogenesis facilitating perineural invasion [18]. This experimental study suggests the existence of the galanin-tumor crosstalk (or generally neuropeptide(s)-tumor interactions) that may possibly play a role in the progression of some cancer types, including colorectal cancer. In this way, galanin could not only be a neuroprotective factor, but it could also play an important role in cancer progression.

In summary, our study showed that the myenteric plexuses' atrophy in the vicinity of cancer invasion was probably not caused by apoptosis or necrosis. Further studies, based on the demonstration of neuropeptides' presence in ENS nerve fibers surrounding tumor invasion in the colon wall and/or enteric neurons' interactions with enteric glial cells may shed new light on the role of ENS-tumor interactions in the progression of colorectal cancer.

\section{Acknowledgements}

This study was supported by the statutory grant No. 25.610.001-300, Faculty of Medical Sciences, the University of Warmia and Mazury in Olsztyn, Poland.

The authors wish to thank Ewa Wedrowska, PhD, for performing statistical analyses of the study and Krzysztof Wasowicz, PhD, DVM, for consulting the manuscript.

\section{References}

1. Timmermans JP, Adriaensen D, Cornelissen W, Scheuermann DW. Structural organization and neuropeptide distribution in the mammalian enteric nervous system, with special attention to those components involved in mucosal reflexes. Comp Biochem Physiol A Physiol. 1997;118:331-340. doi: 10.1016/S0300-9629(96)00314-3.

2. Burleigh DE, Furness JB. Distribution and actions of galanin and vasoactive intestinal peptide in the human colon. Neuropeptides. 1990;16:77-82. doi: 10.1016/0143-4179(90)90115-F.

3. Lang R, Gundlach AL, Holmes FE et al. Physiology, signaling, and pharmacology of galanin peptides and receptors: Three decades of emerging diversity. Pharmacol Rev. 2015;67:118-175. doi: 10.1124/pr.112.006536.

4. Kwiatkowski P, Godlewski J, Kieżun J, Kraziński BE, KmiećZ. Increased galanin serum concentration in colorectal cancer patients results from its production by cancer tissue. Oncol Lett. 2016. [accepted for publication]
5. Pidsudko Z, Kaleczyc J, Wasowicz K et al. Distribution and chemical coding of intramural neurons in the porcine ileum during proliferative enteropathy.J Comp Pathol. 2008;138:23-31. doi:10.1016/j.jcpa.2007.09.003.

6. Skobowiat C, Calka J, Majewski M. Axotomy induced changes in neuronal plasticity of sympathetic chain ganglia (SChG) neurons supplying descending colon in the pig. Exp Mol Pathol. 2011;90:13-18. doi: 10.1016/j.yexmp.2010.11.004.

7. Gonkowski S, Burliński P, Skobowiat C, Majewski M, Całka J. Inflammation- and axotomy-induced changes in galanin-like immunoreactive (GAL-LI) nerve structures in the porcine descending colon. Acta Vet Hung. 2010;58:91-103. doi: 10.1556/ AVet.58.2010.1.10.

8. Elliott-Hunt CR, Pope RJ, Vanderplank P, Wynick D. Activation of the galanin receptor 2(GalR2) protects the hippocampus from neuronal damage. J Neurochem. 2007;100:780-789. doi:10.1111/j.1471-4159.2006.04239.x.

9. Liu M, Song W, Li P et al. Galanin protects against nerve injury after shear stress in primary cultured rat cortical neurons. PLoS One. 2013;8:e63473. doi: 10.1371/journal.pone.0063473.

10. Mansouri S, Barde S, Ortsäter $\mathrm{H}$ et al. GalR3 activation promotes adult neural stem cell survival in response to a diabetic milieu.J Neurochem. 2013;127:209-220. doi: 10.1111/jnc.12396.

11. Godlewski J. Morphological changes in the enteric nervous system caused by carcinoma of the human large intestine. Folia Histochem et Cytobiol. 2010;48:157-162. doi: 10.2478/ v10042-010-0029-8.

12. Macchi B, Marino-Merlo F, Nocentini U et al. Role of inflammation and apoptosis in multiple sclerosis: Comparative analysis between the periphery and the central nervous system. J Neuroimmunol. 2015;15:80-87. doi: 10.1016/j. jneuroim.2015.08.016.

13. Bassotti G, Villanacci V, Maurer CA et al. The role of glial cells and apoptosis of enteric neurones in the neuropathology of intractable slow transit constipation. Gut. 2006;55:41-46. doi: 10.1136/gut.2005.073197.

14. McIlwain DR, Berger T, Mak TW. Caspase functions in cell death and disease. Cold Spring Harb Perspect Biol. 2013;1:a008656. doi: 10.1101/cshperspect.a008656.

15. Jana B, Kozłowska A, Koszykowska M, Majewski M. Expression of cyclooxygenase- 2 in the inflammatory changed porcine uterus. Pol J Vet Sci. 2009;12:1-8. PMID: 19459433.

16. Stevenson L, Allen WL, Turkington R et al. Identification of galanin and its receptor GalR1 as novel determinants of resistance to chemotherapy and potential biomarkers in colorectal cancer. Clin Cancer Res. 2012;18:5412-5426. doi: 10.1158/1078-0432.CCR-12-1780.

17. Kanazawa T, Misawa K, Misawa $Y$ et al. Galanin receptor 2 utilizes distinct signaling pathways to suppress cell proliferation and induce apoptosis in HNSCC. Mol Med Rep. 2014;10:1289-1294. doi: 10.3892/mmr.2014.2362.

18. Scanlon CS, Banerjee R, Inglehart RC et al. Galanin modulates the neural niche to favour perineural invasion in head and neck cancer. Nat Commun. 2015;28:6885. doi: 10.1038/ ncomms 7885 . 